\title{
“Epidemiología de la Menopausia en Colombia: Estudio cooperativo"
}

\author{
Coordinación: \#**W. Onatra \\ Bogotá : \#**Sánchez Jacinto@ Acuña Gabriel@*Arango G. Salazar,@** Barón Germán \&*De la Cruz Jairo, \\ \&**Pardo Francisco, \&***Jaramillo Roberto, Ruiz Alfredo \#* Tovar Gabriel, \#***Caliz Oswaldo, \\ Alvarado Ricardo. \\ Regional: *Díaz Israel, Mesa Edgar DG,*Jaimes Hermes***Gómez Gustavo****Fabio Sánchez.
}

\section{RESUMEN}

OBJETIVO: Se realiza una encuesta prospectiva de corte longitunidal para conocer las características de la Menopausia en Instituciones de salud en Colombia.

MATERIALES Y METODOS: En formulario precodificado se lleva a cabo una encuesta con 75 variables investigando la edad de la menopausia, nivel sociodemográfico, factores de riesgo familiar y personal, antecedentes ginecoobstetricios, laboratorio clínico que incluyó Hemoglobina, glicemia pre y post, colesterol, triglicéridos, biopsia endometrial y terapia instaurada.

RESULTADOS: Se tabularon 832 encuestas distribuidas, 716 en Bogotá $(85.9 \%)$ y 117(14.1\%) en capitales de departamento. La edad promedio de la menopausia fue de 52.26+6.4 años para las regiones y para Bogotá 53.8+7.9. La proporción de Regiones y Bogotá respecto a su origen la Urbana fue $84.2 \% / 92.1 \%$, la raza mestiza $56.3 \%$ predominio sobre la Blanca $42.0 \% / 38.5 \%$ y negra $1.8 \% / 1.2 \%$. El estrato socioeconómico tuvo las siguientes proporciones Medio $55.9 \% / 69.6 \%$, bajo $38.7 \% / 21.9 \%$ y alto $5.4 \%$. Según el estado civil las casadas $\mathbf{6 7 . 0 \% / 6 7 . 6 \%}$ fue mayor que las solteras $\mathbf{1 7 . 4 \% / 1 3 . 3 \%}$. El sintomatología se presentó así: oleadas de calor moderada $56.1 \% / 45.5 \%$, sudoración $52.2 \% / 52.7 \%$, cefalea 50.4\%/41.9\%, Insomnio 40.3\%/40.6\%, Sequedad de piel 44.0\%/36.0\%, Disminución de la libido $25.68 \%$ / $29.6 \%$.

Dentro de los factores de riesgo familiar se encontró como factor predominante la Hipertensión $40 \%$ y la Dislipidemia $13 \%$. Como factores de riesgo personal se encontró el sedentarismo $60 \%$, la Hipertensión $40 \%$ y la ooforectomía $12 \%$. Paraclínicamente la glicemia, el colesterol estuvo dentro de los rasgos normales con un aumento mayor de triglicéridos $160 . .6 \mathrm{mg} / \mathrm{Dd} / 159.7 \%$. La edad de la menopausia es mayor en paciente a mayor número de hijos, y el $50 \%$ recibe terapia hormonal y el 30 no hormonal.

CONCLUSIONES: La edad de la menopausia en este grupo hospitalario es de 52 años, promedio mayor de lo reportado por otros autores, su síntoma principal son las oleadas de calor y sudoración, con factores de riesgo para enfermedad coronaria, datos desconocidos para este grupo etario.

\section{SUMMARY}

OBJECTIVE: A prospective longitudinal inquiry is made in order to know the characteristics of menopause in health Institutions in Colombia.

INSTRUMENTS AND METHODS: A 75 variable inquiry is made on a precodified formulary looking for the menopause age, sociodemographic level, familiar and personal risk factors, gyneco-obstetric antecedents, clinic laboratory including Hemoglobine, pre and post glycemia, cholesterol, triglycerids, endometrial biopsia and instaured therapy.

RESULTS: 832 inquiries were tabulated distributed: 716 in Bogota $(85.9 \%)$ and $117(14.1 \%)$ in departments'head cities. The average age of menopause was $52.26+6.4$ years old in regions and 53.8 + 7.9 in Bogota. The proportion of regions and Bogota according to their origin was: Urban $84.2 \% / 92.1 \%$; Mestizo race $56.3 \%$, prevailing over White $42.0 \% / 38.5 \%$, and Black $1.8 \% / 1.2 \%$. The socioeconomical layer had the following proportions: Middle 55.9\%/69.6\%, Lower $38.7 \% / 21.9 \%$ and High $5.4 \%$. As for the civil state, married women showed $67.0 \% /$ $67.6 \%$; higher than single women, $17.4 \% / 13.3 \%$. Symptomatology was presented like this: moderate warmth surges $56.1 \% / 45.5 \%$, sweating $52.2 \% / 52.7 \%$, cefalea $50.4 \% / 41.9 \%$, insomnia $40.3 \% / 36.0 \%$, libidous decrease $25.68 \% / 29.6 \%$.

@ Instituto de Menopausia

@* Hospital San Rafael.

@** Hospital Universitario Lorencita Villegas de Santos.

\&* Escuela Militar de Medicina. Hospital Militar Central.

\&** Universidad Javeriana. Hospital San Ignacio.

\&*** Universidad del Rosario. Hospital San José

\#* Instituto Seguros Sociales. Clínica San Pedro Claver.

\footnotetext{
\#** Universidad Nacional de Colombia. Instituto Materno Infantil. \#*** Epidemiólogos

* Asociación de Menopausia del Atlántico. Barranquilla

** Universidad Industrial de Santander. Hospital Ramón González Valencia.

*** Universidad del Valle. Hospital Universitario del Valle.

**** Universidad de Antioquia. Hospital San Vicente de Paul.
} 
Among the family risk factors Hypertension $40 \%$ and Dislipidemy $13 \%$ were found as main agents. As personal risk agents, sedentary $60 \%$, Hypertension $40 \%$, and ooforectomy $12 \%$. Paraclinically glycemia; cholesterol was among the normal features with a higher increase in triglycerids $160 . .6 \mathrm{mg} / \mathrm{Dd} / 159.7 \%$. Menopause age is higher in patients with a high children number, and $50 \%$ receives hormonal therapy and $30 \%$ no-hormonal therapy.

Conclusions: Menopause age in this hospitalary group is 52 years old, a higher average than the one reported by other authors; its main symptom is warmth surges and sweating, with risk factors for coronary disease, unknown date for this age group.

\section{Introducción}

La menopausia entendida como el cese definitivo de la menstruación por pérdida de la función ovárica y el Climaterio como un período de transición entre la vida reproductiva y la senectud, han sido aceptados por la mayoría de los investigadores en el tema. Se han clasificado también 3 períodos: Premenopausia entre los 40-50 años, Perimenopausia 3-5 alrededor de la menopausia y la Postmenopausia entre los 50-60 años $(1,2)$.

La edad de la menopausia ha permanecido estable alrededor de los 50 años desde el siglo VI D.C. (3), con algunas variaciones según país, raza y condiciones socioeconómicas. En Estados Unidos es de 51.4, Noruega 41.7, y Nueva Guinea en el Africa 43.6 años (4). En nuestro medio hay dos estudios con promedio de $47.0 \mathrm{y}$ 48.6 años $(5,6)$. Cuando la menstruación se suspende antes de los 40 años (para otros autores antes de los 35 años) es clasificada como Falla Ovárica Prematura (FOP) antigüamente llamada «menopausia prematura o precoz». Existe además un aumento progresivo a partir de los 22 años de la extirpación quirúrgica del útero y ovarios por causas infecciosas o tumorales con cifras americanas de 30\% (7). A esta modalidad se le ha asignado el nombre de Menopausia Quirúrgica.

Las diferentes estadísticas muestran cómo la población mundial a pesar de los programas de planificación ha venido en aumento en progresión casi geométrica con 200 millones en 1950,350 'en 1975 , esperando 590' para el año 2000 y 1100 ' para el 2025 (8).

Por otra parte la expectativa de vida ha aumentado en la medida en que las condiciones socioeconómicas mejoran y es así como para los países desarrollados en 1984 era de 65-74 años y para el año 2000 se espera que sea entre los 80-85 años. Para los países en vía de desarrollo era de 60 años para 1980 esperándose para el año 2000 de 75 años (9). Para Colombia los datos muestran que el promedio de vida en los años 50 era de 55 años, 1980, 62 años, para el 2000 de 78 (10). La proporción de mujeres menopáusicas se ha incrementado aceleradamente y se espera para el año 2000 sea de 6'500.000.

Dentro de diferentes factores atribuidos para explicar la menopausia se destacan: a) el factor genético observándose una edad aproximada en la misma familia (11) o la falla ovárica prematura encontradas en las disgenesias gonadales tipo Turner donde es sabido su alteración cromosómica (12). b) Los datos históricos muestran que la edad no ha cambiado en las últimas dos centurias, con un promedio a los 50 años (13). c) La raza y el área geográfica deben tener una edad más temprana (46 años) que las nórdicas (48 años) y mediterráneas (50 años) (14), se sabe además que la menopausia aparece 1-1.5 años antes en las mujeres que viven por encima de los 2.000 $\mathrm{mts}(15)$. d) Es más temprana la menopausia en las que no han tenido relaciones sexuales regulares que en las casadas (16) así como a un mayor número de gestaciones la menopausia es más tardía (17). e) Otra asociación interesante ha sido relacionada con las mujeres infértiles y oligomenorréicas, presentándose su menopausia un poco más temprano que el promedio y no existiendo relación entre las edades de la menarquia y menopausia (18). f) Las mujeres que realizan trabajos agotadores presentan menopausia más temprana, especulándose sobre el papel de los neurotransmisores a nivel hipotalámico (19). g) Durante la vida reproductiva se sabe también que el ejercicio excesivo en especial en mujeres atletas produce una hiperprolactinemia relativa con amenorrea y falla ovárica prematura, reflejada posteriormente con un adelanto de la menopausia (20). h) Al demostrarse algunos factores autoinmunes en el ovario, asociado a enfermedades sistémicas que cursan con falla ovárica podría ser en parte la razón etiológica del adelanto de la menopausia (21). i) No se ha podido demostrar que los ingresos, la educación y en general el nivel socioeconómico estén relacionados con la edad de la menopausia (22) los estados de desnutrición si presentan un adelanto de la misma. j) Los diferentes estudios sobre anticoncepción y menopausia son controversiales pues para algunos lleva a la atresia folicular (2) y para otros detiene el desarrollo, preservando su función sin afectar la edad de la menopausia (4). k) En las grandes fumadoras, más de un a dos paquetes de cigarrillos al día, con una duración mayor de 10 años se ha demostrado que la menopausia se presenta más temprana atribuida al espasmo vascular tanto ovárico como hipotalámico (23). 1) La extracción quirúrgica del útero también ha demostrado su adelanto atribuido a una insuficiencia vascular (24).

\section{Material y Métodos}

Aprovechando la consulta existente sobre el Climaterio en el país, en los hospitales universitarios se decide por parte del grupo de Climaterio, organizado en el XX Congreso de Obstetricia y Ginecología en Barranquilla conocer el comportamiento epidemiológico de la Menopausia en este grupo etario. El estudio fue prospectivo de corte transversal de las mujeres que consultaron entre el 1 de marzo de 1992 y el 30 de octubre de 1993, por oleadas de calor, síntoma climaterios o trastornos del ciclo, con edades entre 40 y 65 años. Se incluyeron los casos de falla ovárica prematura y las ooforectomizadas, 
por debajo de los 40 años. El formulario único contempló las siguientes variables demográficas: edad, origen, estado civil, estrato socioeconómico, principales motivos de consulta, factores de riesgo familiar y personal, antecedentes ginecoobstétricos, examen colesterol, triglicéridos, HDL, Rx de columna, mamografía, Densitometría, citología vaginal, biopsia endometrial y terapia establecida.

La tabulación se procesó en el programa de base de datos Dbase III, ephiinfo 5.1. y Tru epistat 2.0. Como pruebas estadísticas se empleó la $\mathrm{T}$ de student para observar las diferencias de los promedios y el Chicuadrado para las diferencias entre las proporciones.

\section{Resultados}

La muestra incluyó 832 mujeres climatéricas distribuidas así: 716 en Bogotá y 117 en el resto del país como lo demuestra la tabla 1 .

\section{Tabla 1}

\section{DISTRIBUCION DE LA ENCUESTA POR CIUDADES}

\begin{tabular}{|lcr|}
\hline Ciudad & No. de Encuestas & $\%$ \\
\hline Bogotá & 716 & 85.90 \\
Barranquilla & 33 & 3.96 \\
Medellín & 23 & 2.76 \\
Bucaramanga & 22 & 2.64 \\
Cali & 20 & 2.40 \\
Otras & 18 & 2.16 \\
Total & 832 & 100.0 \\
\hline
\end{tabular}

La menopausia Fisiológica representa el 63\% seguida de forma quirúrgica en un $27 \%$ y precoz $5 \%$. Tabla 2.

Tabla 2

CLASIFICACION DE LA MENOPAUSIA EN \%

\begin{tabular}{|lcc|}
\hline Tipo de Menopausia & Bogotá & Regiones \\
\hline Fisiológica & 65.2 & 61.0 \\
Quirúrgica & 27.1 & 27.6 \\
Precoz & 5.1 & 5.7 \\
Sin Clasificar & 2.5 & 4.8 \\
\hline
\end{tabular}

La edad de la Menopausia en promedio fue de 52.26 años con la siguiente distribución por ciudades. Tabla 3 .

Tabla 3

EDAD PROMEDIO DE LA MENOPAUSIA

\begin{tabular}{|lcc|}
\hline Ciudades & X Edad & D.S. \\
\hline Bogotá & 53.0 & 7.9 \\
Barranquilla & 52.2 & 5.8 \\
Bucaramanga & 46.7 & 8.1 \\
Cali & 52.1 & 4.6 \\
Medellín & 57.3 & 7.4 \\
\hline
\end{tabular}

Las características sociodemográficas se presentan enl la Tabla 4.

Tabla 4

CARACTERISTICAS SOCIODEMOGRAFICAS \%

\begin{tabular}{|lrr|}
\hline Procedencia & Bogotá & Regiones \\
Urbana & 92.1 & 84.2 \\
Rural & 7.8 & 15.8 \\
Estado Civil & & \\
Casada & 67.6 & 67.0 \\
Solteras & 13.3 & 17.4 \\
Viudas & 8.8 & 8.7 \\
U. Libre & 9.9 & 6.9 \\
Raza & & \\
Mestiza & 60.2 & 56.3 \\
Blanca & 38.5 & 42.0 \\
Negra & 1.2 & 1.8 \\
Estrato Socioeconómico & \\
Bajo & 21.9 & 38.7 \\
Medio & 69.6 & 55.9 \\
Alto & 8.3 & 5.4 \\
\hline
\end{tabular}

Los principales síntomas como motivos de Consulta en el momento del ingreso se muestra en la Tabla 5.

Tabla 5

MOTIVOS DE CONSULTA \%

\begin{tabular}{|lrr|}
\hline Síntomas & Bogotá & Regiones \\
\hline Oleadas de calor & & \\
$\quad$ Ausente & 5.3 & 1.2 \\
Leve & 17.3 & 14.6 \\
$\quad$ Moderada & 45.5 & 56.1 \\
$\quad$ Severa & 31.6 & 28.0 \\
Frecuencia & & \\
$\quad>5$ por día & 36.2 & 48.3 \\
$\quad$ < 5 por día & 12.1 & 4.3 \\
Sudoración & 52.7 & 52.2 \\
Cefalea & 41.89 & 50.45 \\
Insomnio & 40.64 & 40.36 \\
Sequedad de Piel & 36.03 & 44.0 \\
Disminución libido & 29.60 & 25.68 \\
Depresión & 20.7 & 10.2 \\
Sequedad de Piel & 18.4 & 7.5 \\
Dolor Lumbar & 17.7 & 20.18 \\
Ansiedad & 17.5 & 10.5 \\
Adinamia & 11.73 & 25.68 \\
Alt. Ciclo & 9.07 & 9.17 \\
\hline
\end{tabular}

Dentro de los factores de riesgo tanto familiar como personal se destacan: Tablas 6 y 7 . 
Tabla 6

FACTORES DE RIESGO FAMILIAR \%

\begin{tabular}{|lrc|}
\hline Factor & Bogotá & Regiones \\
\hline Hipertensión & 40.0 & 40.0 \\
Dislipidemias & 12.9 & 13.6 \\
Cáncer & 10.5 & 16.0 \\
A. C. Vascular & 11.6 & 10.0 \\
Diabetes & 5.0 & 4.7 \\
\hline
\end{tabular}

Tabla 7

FACTORES DE RIESGO PERSONAL \%

\begin{tabular}{|lrc|}
\hline Factor & Bogotá & Regiones \\
\hline Hipertensión & 40.0 & 40.0 \\
Ooforectomía & 10.89 & 13.76 \\
* Tabaquismo & 11.6 & 6.0 \\
Alcohol & 6.3 & 4.5 \\
** Fármacos & 4.8 & 6.0 \\
Hiperlipidemia & 4.3 & 12.0 \\
Actividad Física & & \\
Leve-Ninguna & 57.7 & 21.3 \\
Moderada & 21.6 & 66.0 \\
Intensa & 1.5 & 8.5 \\
\hline
\end{tabular}

* El promedio de cigarrillo y la duración para Bogotá fue de 6.22 x 13.18 años y para las Regiones de $9.0 \times 8.17$ años.

**El consumo de antihipertensivos era de $31.49 \%$ y anti-tiroideos del $10.2 \%$

Los antecedentes Gineco-obstétricos se muestran en la Tabla 8 y la relación de Menarquia y Número de gestaciones en relación con la edad de la Menopausia en la Tabla 9 .
Los datos del Examen Físico se muestran en la Tabla 10 .

Los exámenes paraclínicos de química sanguínea se muestran en la tabla 11 . Los datos de radiografía de columna lumbar fueron inconsistentes y los de la Densitometría se presentan en un trabajo aparte dado que hasta ahora se ha iniciado la tabulación a nivel regional. Los datos de biopsia endometrial sólo aparecen 72 muestras para Bogotá.

Los tratamientos establecidos se presentan en la Tabla 12.

\section{Discusión}

La menopausia entendida como un período transcisional en la vida de la mujer entre su época reproductiva y no reproductiva caracterizada por la desaparición de la menstruación no puede quedarse en una forma simplista y biológica. Este período marca no sólo un cambio hormonal sino todas las repercusiones en la economía del organismo a nivel cardiovascular, óseo y metabólico. Al lado de la sintomatología clínica se debe tener en cuenta el patrón sociocultural, económico y psicoafectivo donde se desenvuelva la mujer, porque de estos factores se desprende la negación, exageración o aceptación de este evento.

Tabla 8

ANTECEDENTES GINECOOBSTETRICOS

\begin{tabular}{|lcc|}
\hline Antecedentes & Bogotá & Regiones \\
\hline Menarquia & 13.65 & 13.25 \\
Gestaciones & 4.28 & 4.73 \\
Partos & 3.69 & 4.08 \\
Abortos & 1.77 & 1.64 \\
\hline
\end{tabular}

Tabla 9

COMPARACION ENTRE LA EDAD DE LA MENARQUIA NUMERO DE GESTACIONES FRENTE A LA EDAD DE LA MENOPAUSIA

\begin{tabular}{|ccccrc|}
\hline $\begin{array}{c}\text { Menarquia } \\
\text { (años) }\end{array}$ & $\mathrm{n}$ & $\begin{array}{c}\text { X edad } \\
\text { Menopausia }\end{array}$ & Gestaciones & $\mathrm{n}$ & $\begin{array}{c}\text { X edad } \\
\text { Menopausia }\end{array}$ \\
\hline$<10$ & 14 & 50.0 & 0 & 4 & 56.25 \\
11 & 44 & 51.29 & 1 & 29 & 49.24 \\
12 & 99 & 51.82 & 2 & 89 & 49.91 \\
13 & 133 & 50.66 & 3 & 103 & 50.91 \\
14 & 123 & 51.84 & 4 & 108 & 50.97 \\
15 & 98 & 52.43 & 5 & 67 & 52.12 \\
16 & 40 & 53.15 & 6 & 41 & 53.61 \\
17 & 24 & 50.00 & 7 & 22 & 52.27 \\
$>17$ & 14 & $50.28 *$ & 8 & 15 & 54.53 \\
& & & $>8$ & 49 & $56.52 * *$ \\
\hline
\end{tabular}

* No significativa

** Prueba significativa 
Tabla 10

EXAMEN FISICO

\begin{tabular}{|lrr|}
\hline Categorías & Bogotá & Regiones \\
\hline Peso Kg & 62.0 & 65.05 \\
Talla cm. & 1.57 & 1.53 \\
I. Masa Corporal & 25.2 & 27.8 \\
Tensión Arterial & & \\
$\quad$ Sistólica mmHg & & \\
$<140$ & 56.68 & 63.71 \\
140-160 & 32.89 & 29.20 \\
$>160$ & 6.75 & 6.19 \\
Diastólica mmHg & & \\
$<90$ & 61.67 & 66.37 \\
$90-105$ & 31.42 & 29.20 \\
$>105$ & 3.23 & 2.65 \\
\hline
\end{tabular}

Tabla 11

EXAMENES PARACLINICOS \%

\begin{tabular}{|lrr|}
\hline Parámetro & Bogotá & Regiones \\
\hline Hemoglobina & 13.85 & 12.13 \\
Glicemia pre & 88.9 & 82.72 \\
Glicemia post & 91.14 & 90.77 \\
Colesterol & 222.95 & 210.65 \\
Triglicéridos & 159.72 & 160.68 \\
HDL & 48.31 & 55.62 \\
Biopsia Endometrial* & \\
Secretor & 3.63 & \\
Proliferativo & 4.46 & \\
Hiperplasia & 0.90 & \\
Ca. Endometrial & 0.90 & \\
\hline
\end{tabular}

* Datos no tabulables por el número escaso $n=72$

Tabla 12

TRATAMIENTOS ESTABLECIDOS

\begin{tabular}{|lcc|}
\hline Tratamiento & Bogotá & Regiones \\
\hline Hormonal & 45.81 & 55.75 \\
E.solos & 25.30 & 21.90 \\
E+Progest. & 36.00 & 56.30 \\
& & \\
No Hormonal & 30.00 & $*$ \\
Ca+Terap Física & 23.80 & \\
Ca+Dieta & 14.5 & \\
Ca+Fluor & 11.9 & \\
Tranquilizantes & 9.80 & \\
\hline
\end{tabular}

* No tabulables
La edad de la menopausia ha permanecido estable a lo largo de este siglo y los promedios están alrededor de los 50 años. La edad de la menopausia en el presente estudio está en 52.26 años un poco por encima de lo reportado por otros estudios hechos en nuestro medio y en otros países. Tabla 13 Se plantea la posibilidad de que este hallazgo coincida con algunos factores raciales, climáticos o medioambientales y la necesidad de ampliar la muestra a la población general para aceptar que en nuestro medio la edad de la menopausia es mayor que en otros países.

- La encuesta sociodemográficamente muestra una población urbana en un 80-90\% con escasa representación del sector rural, datos compartidos con otros investigadores $(15,25)$ y donde se hace necesario realizar un estudio en este sentido con el fin de corroborar o no el cambio de la edad de la menopausia en esta área. Racialmente nuestra población es mestiza y de acuerdo con los estudios realizados por la Sección de Genética de la Universidad Nacional (26) hay predominio de la raza blanca en los santandereanos y de la raza negra en la Costa Atlántica y Pacífica. Diferentes estadísticas mundiales reportan que la edad de la menopausia es más temprana en la raza mediterránea y africana. En el presente estudio en una manera representativa de la población climatérica hospitalaria muestra que la raza mestiza es el $60 \%$ y blanca el $40 \%$, con un promedio de edad de 52 años un poco mayor de lo reportado por otros países. En relación al nivel socioeconómico es difícil hacer inferencias dado el número tan bajo de las pacientes a nivel alto y lo mismo en la proporción de solteras y casadas. Los datos generales informan que la edad de la menopausia es más baja cuando las condiciones socioeconómicas son precarias como en el Africa y la edad se retrasa en las mujeres casadas que en solteras $(2,4,15,27)$.

A pesar de ser un hecho biológico y de existir un desencadenante único como factor etiológico en la menopausia -disminución de los estrógenos circulantes- es un hecho comprobado que la sintomatología es muy variable y multifactorial. Explicación aceptada por la acción ejercida por estos a nivel del sistema nervioso central, metabólico, circulatorio, lomotor, piel, genitourinario. Encontrar pacientes asintomáticas (10$15 \%$ ), al lado de otras plurisintomáticas se tiene una gama de síntomas desde leves a severos y de intensidad variable. Ya lo decía Maranon en 1936, «cada mujer realizará un tipo de climaterio diferente con arreglo a su constitución endocrina».

La frecuencia de la sintomatología durante el climaterio varía según los diferentes autores pero en general hay acuerdo de que las oleadas de calor y la sudoración es el primer síntoma de queja. Al comparar estos síntomas con los datos reportados por Lauritzen en Alemania (28), Palacios en España (15) y en el presente estudio observamos que los Insomnios, depresión e irritabilidad son más frecuentes en el estudio alemán, mientras en España son el dolor articular, Insomnio y cefalea, para Colombia en este estudio son la cefalea, Insomnio y la resequedad de piel, es de anotar que el dolor lumbar, la disminución de la libido y la dispareunia ocupan el 20\%. Tabla 14. 
Tabla 13

EDAD DE LA MENOPAUSIA: COMPARATIVO POR PAISES

\begin{tabular}{|c|c|c|c|c|c|}
\hline País & Año & Autor & Raza & Edad & Tipo de estudio \\
\hline Alemania & 1972 & Hofmann & Blanca & 49.6 & retrospectivo \\
\hline \multirow[t]{2}{*}{ Inglaterra } & 1964 & Fromer & & 49.8 & \multirow[t]{2}{*}{ cross-sectinal } \\
\hline & 1972 & McKinley & Blanca & 50.8 & \\
\hline Escocia & 1970 & Thompson & Blanca & 50.1 & cross- \\
\hline \multirow[t]{2}{*}{ USA } & 1966 & McMahon & Negra & 49.3 & \multirow{2}{*}{$\begin{array}{l}\text { cross } \\
\text { cohorte }\end{array}$} \\
\hline & 1974 & Treloar & Blanca & 49.8 & \\
\hline \multirow[t]{2}{*}{ Sudáfrica } & \multirow[t]{2}{*}{1960} & \multirow[t]{2}{*}{ Abrahamson } & Blanca & 48.1 & \multirow{2}{*}{$\begin{array}{l}\text { retrospectivo } \\
\text { retrospectivo }\end{array}$} \\
\hline & & & Negra & 47.7 & \\
\hline \multirow[t]{2}{*}{ España } & 1977 & Botella & Blanca & 50.1 & \multirow{2}{*}{$\begin{array}{l}\text { retrospectivo } \\
\text { retrospectivo }\end{array}$} \\
\hline & 1985 & Caballero & Blanca & 48.6 & \\
\hline Nueva Guinea & 1973 & Gray & Asiática & 47.3 & cross \\
\hline India & 1966 & Wyon & Asiática & 44.0 & retrospectivo \\
\hline \multirow[t]{3}{*}{ Colombia } & 1970 & Luzardo & Mestiza & 47.0 & \multirow{3}{*}{$\begin{array}{l}\text { retrospectivo } \\
\text { prospectivo } \\
\text { prospectivo }\end{array}$} \\
\hline & 1990 & Rojas & Mestiza & 48.6 & \\
\hline & 1994 & Presente & Mestiza & 52.2 & \\
\hline
\end{tabular}

Tabla 14

COMPARATIVA DE SINTOMAS DURANTE EL CLIMATERIO \%

\begin{tabular}{|lccc|}
\hline Síntomas & $\begin{array}{c}\text { Lauritzen (28) } \\
\text { Alemania }\end{array}$ & $\begin{array}{c}\text { Palacios (15) } \\
\text { España }\end{array}$ & $\begin{array}{c}\text { Presente } \\
\text { Estudio }\end{array}$ \\
\hline O de Calor & 69 & 56 & 66 \\
Sudoración & 58 & 45 & 52 \\
Insomnio & 56 & 33 & 40 \\
Depresión & 70 & 11 & 20 \\
Irritabilidad & 49 & 15 & 10 \\
Cefalea & 31 & 19 & 45 \\
Sequedad piel & - & 18 & 38 \\
Dolor articular & - & 37 & 18 \\
Pérdida libido & - & 15 & 27 \\
Dispareunia & - & 19 & 13 \\
\hline
\end{tabular}


Referente a la sexualidad los estudios clásicos de Kinsey en el 53 reportaban una disminución tanto en la frecuencia como el deseo en un $48 \%$ en mujeres postmenopáusicas (29). Posteriormente Pfeiffer en el 72 en una encuesta de 241 mujeres entre los 45 a los 69 años encontró que la disminución del interés era del $7 \%$ en mujeres menores de 30 años, del $20 \%$ entre los $51-55$ y del $31 \%$ entre 56 y 60 años (30). Los datos encontrados en el presente estudio están dentro de los rangos esperados para este grupo de edad. La dispareunia por el contrario oscila entre un $42-48 \%$ atribuida en parte a la resequedad vaginal o disminución del interés de su compañero. (31).

El estudio de Framminghan sobre enfermedades cardíacas demostró que el riesgo llegaba a igualarse en los dos sexos después de 5 a 8 años de la postmenopausia y que el riesgo de infarto es mayor en mujeres postmenopáusicas que premenopáusicas (32). Se sabe también que el riesgo de arterioesclerosis es 4 veces mayor en la postmenopausia que en la premenopausia. Estos hallazgos han postulado el efecto protector de los estrógenos sobre la pared arterial por el aumento de las Lipoproteínas de alta densidad (HDL) y la experimentación animal que demostró en macacas sometidas a dieta rica en colesterol y dosis altas de estrógenos no desarrollaron placas ateromatosas. (33). Dentro de los factores de riesgo cardiovascular se destacan: la edad avanzada, historia familiar de enfermedad coronaria, Hipercolesterolemia, tabaquismo, hipertensión, diabetes mellitus, obesidad o antecedentes de accidente cerebrovascular (ACV) o enfermedad vascular periférica. Por los datos obtenidos en la encuesta se detectaron 4 de los factores de riesgo a nivel familiar (Hipertensión, ACV, Dislipidemia y diabetes) y 4 factores a nivel personal (Hipertensión, sedentarismo, tabaquismo y dislipidemia) implicando que la mujer menopáusica en nuestro medio presenta factores de riesgo algunos de estos prevenibles.

Estos datos contrastan muy bien con los hallazgos epidemiológicos de la poca actividad física de nuestras mujeres y el aumento de los triglicéridos. Se sabe que el riesgo de enfermedad coronaria es proporcional al aumento de colesterol y triglicéridos y que éstos se elevan progresivamente al aumentar la edad (34), pero que disminuyen $3 \%$ por cada $2 \%$ de reducción de colesterol y de HDL. La medición de la relación HDL/LDL son más específios calculándose que un incremento de $1 \mathrm{mg} / \mathrm{dl}$ de Hdl hay un 3-5\% de disminución de enfermedad coronaria (35). Niveles de triglicéridos y HDL menos de $88 \mathrm{mg} / \mathrm{dl}$ y $50 \mathrm{mg} / \mathrm{dl}$ respectivamente están asociados con la disminución de enfermedad coronaria.

Durante la menopausia en endometrio se atrofia, y sus glándulas se reducen y quedan limitadas a la basal, pero conservan su capacidad de reaccionar ante los estrógenos, endógenos o exógenos (36) Berniske observa que el endometrio basal se interdigita con el miometrio con la edad, lo que da como resultado cierto grado de adenomiosis superficial, que es hallazgo normal en el útero durante la quinta década. Este endometrio basal infiltrado no experimenta los cambios cíclicos normales.

Después de la menopausia la atrofia endometrial es evidente y la actividad mitótica cesa. Las células epiteliales disminuyen de tamaño y el estroma se torna fibrótico. Se acumula un material eosinófilo compacto en las glándulas endometriales, que en ocasiones las ingurgita. Esto causa el cuadro histológico de «atrofia quística», que es característico del endometrio postmenopáusico. Estos cambios atróficos pueden ocurrir en las mujeres tratadas con agentes que suprimen la función hipófiso-gonadal, entre ellos los agonistas de la hormona liberadora de gonadotrofinas $(\mathrm{GnRH})$ (37).

Hay informes recientes que refieren una notable tasa de progresión de la hiperplasia endometrial con atipia citológica a carcinoma ( 24 a $57 \%$ de las pacientes. Por el contrario existe una probabilidad relativamente baja de progresión de la hiperplasia simple o quística. Esto ha llevado a algunos investigadores a considerar la hiperplasia con atipia celular como una lesión biológica separada diferenciada de las otras formas histológicas con menor potencia para la transformación maligna por la denominación de «neoplasia intraepitelial endometrial» (38-39).

En este estudio se tomó biopsia de endometrio a 72 mujeres del grupo de menopausia pertenecientes al área de Bogotá y se encontró la siguiente distribución: endometrio secretor $26(36 \%)$, endometrio proliferativo $32(44.4 \%)$, hiperplasia endometrial $6.44(8.9 \%)$ y para el Ca. de endometrio del 6.44 (8.9\%).

Aunque la muestra no es representativa, se deben seguir tomando biopsias para confirmar el comportamiento similar encontrado en la literatura (40-42). Hay una marcada tendencia en los endometrios no atróficos, generalmente proliferativos simples, pero en ocasiones hiperplásicos. Esto es debido a que los niveles de estradiol están todavía muy altos en los primeros años después de la cesación de las reglas. Como al mismo tiempo el cuerpo lúteo del ovario ha desaparecido mucho antes, hay así una acción estrogénica no contrabalanceada por progesterona y hay lo que se ha llamado hiperestrogenismo climatérico. Padwuick y col (43) insisten en la necesidad de tratar estas mujeres no con estrógenos, sino con gestágenos. De Brux da una proporción de hiperplasias postmenopáusicas hasta del $15 \%$, mientras que nosotros encontramos $46 \%$. Gisela Dallenbach-Hellweg cree que se trata de seudohiperplasias determinadas por la senecencia del epitelio. Pero se piensa que no es así, se creen que son verdaderas hiperplasias hiperestrogénicas o mejor dicho producidas por niveles no muy altos de estrógenos, pero en ausencia completa de progesterona. Es sabido que la progesterona deprime los receptores estrogénicos del endometrio (2). Por si fuera poco, Tseng y Guepide (44) han demostrado que la progesterona eleva la estradiol-deshidrogenasa del endometrio, y que la falta de ella, hace que el estradiol se acumule. Tsibris y cols. (45) estudiando los receptores de estradiol y de progesterona en el endometrio humano, describe que al llegar la menopausia los primeros persisten, pero que los segundos desaparecen. Este mecanismo local de los receptores, sería, mucho más que los niveles altos de estradiol circulante, lo que causaría esta elevada tendencia a la hiperplasia del endometrio climatérico. O dicho con otras palabras es la falta de progesterona la principal motora de este cuadro. Esta es una razón decisiva en 
favor de los que aconsejan gastanoterapia en el climaterio. (46)

Claro está que esta hiperplasia es causa de muchas hemorragias postclimatéricas, vemos que un elevado porcentaje de las mujeres con sangrado después de la menopausia tenían alteraciones hiperplásicas en el endometrio. Otra causa de sangrado postmenopáusico, principalmente cáncer de endometrio, deben ser consideradas. Merril encuentra que las hemorragias postmenopáusicas, sólo 8,6\% coinciden con cáncer de endometrio, mientras 6,3 son por hiperplasia. Es curioso que la mayoría de los casos coinciden con atrofia del endometrio, a pesar de cursar con hemorragias, pero debe tenerse en cuenta que se incluyen aquí otras causas, como atrofia vaginal sangrante, que son muy frecuentes y que producen también la apariencia de una metrorragia.

En la mujer postmenopáusica que no se trata con estrógenos, la frecuencia de carcinoma endometrial es de alrededor de 1/1000 mujeres/año. En varios estudios se demostró que la relación entre el carcinoma endometrial y la administración de estrógenos exógenos (36). No hay duda que esta relación depende de la dosis y el tiempo en que se utiliza. En el estudio de Duke, Hammond y cols encontraron que el riesgo de carcinoma de endometrio aumenta cuando la dosis es de 0.625 o más de estrógenos conjugados administrados durante al menos cinco años. En este estudio, la administración de estrógenos cíclicos no protegía contra el carcinoma, mientras que la adición de un progestágeno sí lo hacía.

En el estudio de Rosenwaks y cols los progestágenos no parecieron proteger contra el carcinoma ni la hiperplasia endometrial. Esta diferencia en los estudios, puede indicar que la dosis y la duración del progestágeno es crítica. Rosewaks no logró predecir el aspecto del endometrio con base en el cuadro hemorrágico. Los patrones con hiperplasia grave en ocasiones no presentan hemorragia regular por supresión o hemorragia irregular. La única paciente que desarrolló cáncer endometrial en el grupo demostró hemorragia regular por supresión. Esta hemorragia por supresión de progestágenos no se acompaña de descamación completa de la capa endometrial funcional. Por lo tanto no protege contra la aparición de cáncer endometrial (38-39).

Hay varios estudios retrospectivos en donde se indica el riesgo de adenocarcinomas de endometrio en pacientes tratadas con estrógenos aumenta de 1.7 a 20 veces. La adición de algún progestágeno cíclico al esquema de estrógenos proporciona un efecto protector contra el cáncer endometrial. Existe un riesgo de tres a nueve veces mayor de cáncer endometrial en la mujer postmenopáusica que tiene un sobrepeso de 12 a $25 \mathrm{~kg}$ (112). A diferencia de los estudios retrospectivos en los que se informa un mayor riesgo de cáncer endometrial en usuarias de estrógenos en las postmenopausias en el Wilford Hall United States Air force Medical Center se observó una reducción constante no obstante en el uso de estrógenos y progestágenos (44).

Estas conclusiones sugieren que el suministro de estrógenos aumenta en forma considerable la frecuencia de cáncer endometrial, pero no la mortalidad. Los estrógenos cíclicos no constituyen menor riesgo si su administración es continua. La adición de un progestano proporciona una considerable protección. De ahí la importancia de antes de comenzar el tratamiento con estrógenos, se debe realizar una evaluación completa de la paciente, por medio de una historia clínica minuciosa y un examen físico con estudios de laboratorios apropiados para detectar cualquier factor que pueda colocar a la paciente en un riesgo adverso mayor. Cualquier antecedente de hemorragia anormal requiere una evaluación histológica del endometrio antes de la iniciación del tratamiento con estrógenos y muchos autores recomiendan una evaluación endometrial en todas las pacientes asintomáticas.

Una creencia dentro de la población es que a menarquia temprana la menopausia también es temprana. los diferentes estudios epidemiológicos no han podido demostrar estas hipótesis y por el contrario a menarquia temprana la edad de la menopausia permanece en su promedio. Esta investigación demuestra estadísticamente que a mayor número de hijos la edad de la menopausia es más tardía y está de acuerdo con lo observado también por otros autores $(2,18,27)$.

La investigación sobre el tipo de tratamiento instaurado se observa que un $50 \%$ inicia con terapia hormonal de sustitución y un $30 \%$ en la forma no hormonal. Estos datos están de acuerdo con una encuesta hecha en Bélgica en un total de 647 mujeres menopáusicas donde reciben THS en un $54 \%$ prescrita por ginecólogos (25)

\section{BIBLIOGRAFIA}

1. Studd JWW, Whitehead MI. The Menopause. Blackell Scientific Publications. Oxford 1988;102-115.

2. Botella-Llusia J. La edad crítica. Epidemiología del Climaterios. Edit Salvat. Barcelona España 1990; 51-61.

3. Utian WH. Menopause in modern perspective. A guide to clinical practice. New York. Apleton Century Crofts 1980.

4. Gray RH. The Menopause epidemiological and demographic considerations. In the Menopause, Beard RJ. Edit MPT press Lancaster. $1976 ; 25-40$.

5. Luzardo MF. Pérez G. Climaterio y Menopausia. Rev. Col. Obstet. Ginecol. 1970; 21: 273-315.
6. Rojas J. Onatra W. Edad de la Menopausia y su relación con la menarquia, gestaciones, hábito de fumar y uso de anticonceptivos en el IMI y HSJD de Santafé de Bogotá. Rev. Col. Obstet. Ginecol. 1993; 44: 231-235.

7. Dawood MY, Tidey GF. Menopause. Curr Probl Obstet. Gynecol. \& Fertil 1993; 16(5): 169-208.

8. Koster A. Change-of-life anticipations, attitudes and experiences among middle.age Danish women. Haelth Care Women Int 1991; 12: $1-4$.

9. Diczfalusy E. Menopause, developing countries and the 21 st century. Acta Obstet. Gynecol. Scand Suppl 1986; 134: 45-47. 
10. DANE 1985.

11. Weg RB Demography. In Mishell DR jr. ed. Menopause: physiology and pharmacology. Chicago: Year Boock Medical Publishers; 1987; 23-40.

12. Villanueva AL., Revar RW. Triple «XXX» Syndrome and premature ovarian failure. Obstet. Gynecol. 1983; 60: 705-715.

13. Edwards RG. The Menopause. Research in Reproduction. 1978; 10: 1-3.

14. Walker ARP., Walker BF., Ncongwane J et al. Age of menopause in black women in South Africa. Br. J. Obstet. Gynecol. 1984; 91: 797 801.

15. Palacios S. Climaterio y Menopausia. edit Mirpal, Madrid 1993; 9-15.

16. Hauser GA., Remen V., Valaer M et al. Menarche and Menopause in Israel. Gynecology 1963; 155: 39-47.

17. McKinley S., Jenfferies M., Thompson B. An investigation of the age of the menopause. J Biosoc Sci 1972; 4: 161-173.

18. Treloar AE. Menarche, Menopause and Intervenig Fecundability Human Biol 1974; 46: 89-98.

19. Maoz B., Antonowsky A., Apter A et al. The effect of the outside work in the menopausal women. Maturitas 1978; 1: 43-45.

20. Coulam CB, Adamson SC, Annaegers JF. Incidence of premature ovarian failure. Obstet. Gynecol. 1986; 67: 604-608.

21. Barbo DM. The physiology of menopause. Med. Clin. North. Am. 1978; 71: 13-38.

22. McKinlay SM., Brambilla DJ., Posner JG. The normal menopause transition. Maturitas 1992; 14: 102-105.

23. Miggette AS, Baron JA. Cigarrete smoking and the risk of natural menopause. Epidemiology 1990; 1: 474-476.

24 Siddle N., Sarret P., Whitehead M. The effect of hysterectomy on the age at ovarian failure; identification of a subgroup of women with premature loss of ovarian function and literature review. Fertil Steril 1987; 47: 94-109.

25. Gaspard U. Diagnosis and treatment of the menopause in Belgium. Current Perspectives on hormonal treatment during the menopause. Int Clin Pract Series 1992; 9-18.

26. Ramos O., Yunis ET., Yunis JJ. Aplicaciones de la genética molecular en la práctica forense. Rev. Fac. Med. UN. Col. 1993; 41: 191-199.

27. Khaw KT. Epidemiology of the menopause: Brit Med Bull 1992; 48: 249-261.

28. Lauritzen C. La menopause et son traitement. PIL. 1982.

29. Kinsey AC., Pomeroy WB Et al. Sexual behavior in the human female. WB Saunders 1953; 734-736.
30. Pfiffer E., Verwoerdt A., Davis GC. Sexual behavior in middle life. Am J Psychiat 1972; 128: 1262-1267.

31. Sarrel MP. Sexuality in the Menopause. Studd JWW, Blackwell Sci Publ. Oxford 1988; 65-75.

32. Kannel WB., Hjortlan MC et al. Menopause and risk Cardiovascular disease: The framingham study. Ann. Inter. Med. 1976; 85: 447-452.

33. Barrent-Connor E., Bush T. Estrogen and coronary Heart Disease in women. JAMA 1991; 265: 1861-1867.

34. Heiss G., Tamir I et al. Lipoprotein-Cholesterol distributions in select north american populations: The lipid research clinics program prevalence the studi Circulation 1980; 61:302-315.

35. Lobo RA. Treatment of the postmenopausal woman. Raven Press New York 1994; 199-221.

36. Sherman AL., Brows S. The precursor of endometrial carcinoma. Am. J. Obstetric. Gynecol. 1979; 135: 947.

37. Benirscchke K, Endometrium. In Yen SSC, Jaffe RB Reproductive Endocrinology. Philadelphia, WB saundersco. 1986.

38. Strauss III J.F Gurpide E. Endometrio: regulaclón y disfunción. Yen/ jaffe. Endocrinología de la reproducción. 3a. Edición 1993; 333-403.

39. Kurman RJ and cols, the behavior of endometrial Hyperplasia: Along Term study of untrated hiperplasia in 170 patients. Cancer. 1985; 56: 403.

40. Aiman J. Age, estrogen and endometrium. Clin. Obstet. Ginecol. 1980; 143: 375-380.

41. Botella Llusia J. Cambios en el aparato genital durante el climaterio. La edad crítica: Climaterio y Menopausia. 1990; 85-95.

42. Choo YC. y Cols. Postmenopausal uterine bleeding of non organic cause. Obstet. Gynecol. 1985; 66: 225-230.

43. Padwich ML. y Cols. A simple method for determining the optimal dosage of progesti in postmenopausal women receiving estrogen. $\mathrm{N}$. Engl. J. Med. 1986; 315: 930-934.

44. Tseng L y Gurpide E. Effect of progestins on estradiol receptor levels in human endometrium. J. Clin. Endocrinol. Metab. 1975; 97: 825830.

45. Tsibris JCM, y cols. Distribution of cytoplasmic estrogen and progesterone receptors in human endometrium. Am. J. Obstet. Gynecol. 1978; 132: 449-454.

46. Norris H. and Cols. Lesiones Preinvasoras del Endometrio. Ginecología y Obstetricia. Temas Actuales. 1986; 4: 735-750. 